\title{
Heating the Compact Ignition Tokamak (CIT)
}

\author{
D. W. Ignat \\ Princeton Plasma Physics Laboratory \\ Princeton, New Jersey 08543
}

\begin{abstract}
The proposed CIT starts operation in the late 1990's with $20 \mathrm{MW}$ of of heatizg power. The tokamak and facility are to be designed to accommodate $50 \mathrm{MW}$ auxiliary heating. The heating methods now being considered are ion cyclotron heating (ICH) and election cyclotron heating (ECH). Aspects of these sys?ems are described, and the choice of powet level and type is discussed.
\end{abstract}

\section{DISCLAIMER}

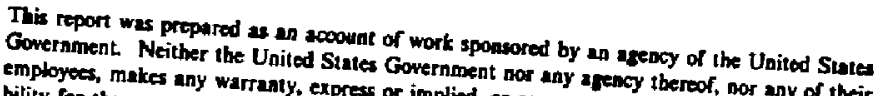
bility for the acsuracy, conjoleterese, or usefulned, or assumes any lesal liability or responiprocess disclowed, or represents that its use would of any information, npss'ratus, product, or manuferein to any specific commercial product, procen infrigte privalely owned rights. Refer. meadation, or favarinerwie does not necesturily constitute or imply trede name, trademarth and opinions of anter by the United States Government or imply its endorsement, recom. Uniled State of authors expreaced herein do not necest of any asency thereof. The views Uniled States Goverament or any meency thereof. 


\section{Requirements}

The CIT is proposed to demonstrate and study ignition prior to the development of a prototype reactor. A small major radius $(2.1 \mathrm{~m})$ allows the cost to remain moderate, while high field and current ( $11 \mathrm{~T}$ and $12.3 \mathrm{MA}$ ) produ $=$ good confinement of plasma energy.' Two essential questions concern the pt e length and the amount of power.

\subsection{Frolse length}

At minimum, the heating power must last for the duration of the maximum magnetic field, which is 5 seconds. This time is selected to be an order of magnitude greater than the energy confinement time $t_{E}$ required for the ignition of a plasme at the empirical density limit of $\approx 5 \cdot 10^{30} \mathrm{~m}^{-3}$. A longer pulse lengti is desired to allow for heating during ramp-up and ramp-down of the magn - ic field. Heating as the field increases adds energy, and temperature, to e plasma before the flat-top periodi and heating as the field returns to zero can possibly help avoid disruptions. Another factor is that the allowable pulse length increases rapidly as the operating field is reduced from the design value. Therefore, operating below maximum field benefits from a longer heating pulse length up to the point of straining the thermal properties of the tokamak and the heating system.

In sum, a practical judgement is that 10 seconds sho ild be the maximum duration for heating.

\subsection{Heating power}

Empirical scaling laws relating election density $n_{e}$, temperature $T_{e, i}$, energy coufinement time $\tau_{E}$, heating power $P_{\text {axx }}$, and other parameters have been the subject of several studies. 1,2 A key premise is that parameters which fit existing tokamak achievements of $\tau_{E}$ can be applied to the fulure, and that an enhancement factor $c_{\tau}$ can be introduced on $\tau_{E}$ to take account of improvements to confinement seen in some discharges. The value of $c_{\boldsymbol{r}}$ is in the range $1.5-2.5$.

The following conclusions can be drawn from confinement studies:

1. Heating power of $20 \mathrm{MW}$ transmitted from an if launcher is sufficient to bring the discharge to ignition early in the flat-top period if confinement is sufficient to allow ignition. 
2. Heating power of $50 \mathrm{MW}$ or more might be required to reach maximum energy multiplication $Q$ if confinement does not support ignition.

3. Operation without tritium will require power greater than $50 \mathrm{MW}$ to reach the $\beta$-limit at full field because of the lack of $\alpha$-heating.

It seems prudent to plan on a range of heating powers, starting with a moderale level for the first discharges and allowing for additions in the future. The initial complement of auxiliary power for CIT is $20 \mathrm{MW}$ based on the first conclusion. The tokamak and supporting facilities will be designed to accommodate up to $50 \mathrm{MW}$ of $\mathrm{rf}$ heating power for high- $Q$ studies with tritium, and high- $n_{e}, T, \beta$ studies withoul tritium.

\subsection{Other requirements}

The basic requirements on the heating are $20 \mathrm{MW}$, expandable to 40 or 50 MW, 10 seconds duration, as stated above. Other important qualities are:

1. Heating efficiency over a broad range of density and temperature;

2. High power density, reducing the number of ports required;

3. Low impurity generation;

4. Robustness of the wave launcher to disruptions, and fluxes of particies and radiation;

5. Usefultress over a range of operating magnetic fields. The maximum field is $11 \mathrm{~T}$. Operation from 8 to $11 \mathrm{~T}$ is desirable for testing and scaling studies. 


\section{Heating methods}

\subsection{Possible technologies}

The technologies tested for heating on tokamaks include the following, in approximate chronological order: neutral beams (40-100 keV particles), lower hybrid (0.5-5 GHz waves), ion cyclotron (30-100 $\mathrm{MHz}$ waves), electron cyclotron ( $60 \mathrm{GHz}$ waves), and high-harmonic ion cyclotron heating (30-200 MHz waves). ICH and ECH have been chosen for integration with the CIT design.

Neutral beam heating (NBH) has been considered, especially in the past year. ${ }^{3}$ However, this option remains inactive because development of sources, accelerators, neutralizers, and transport is not being supported, and because the beam lines change the conception of the facility, and possibly the tokamak. Ion Bernstein wave heating (IBWH), referred to above as high-harmonic ion cyclotron heating, has an attractive implementation based on fundamentalmode waveguides operating at $250 \mathrm{MHz}$. The waveguides fit into the port, wide edge vertical, and are fed with coaxial lines near the tokamak. IBWH could possibly be considered if high power experiments achieve good results in the future. Lower hybrid waves have had good success heating electrons, and outstanding results with current drive. However, an installation for CIT would need $10 \mathrm{GHz}$, and therefore extremely fine-scale launching structures. Also, there is a fundumental limit to penetration at high temperatures (above $10 \mathrm{keV}$ ) from Landan damping.

\subsection{Choices}

\subsubsection{Baseline}

The choice of ICH was based on success in experiments such as PLT and on the ready avilability of power in the needed frequency range (100 $\mathrm{MHz}$ ). Recent ICH results on $\mathrm{JE}^{\prime} \mathrm{I}^{\mathrm{b}}$ and $\mathrm{TFTR}^{*}$ have demonstrated efficient heating at temperatures relevant to ignition. The plan is to implement a $20 \mathrm{MW}$ ICH system on CIT for first operation, using five ports of the tokamak, and ten transmitters adapted from the former Fusion Materials Irradiation Test (FMI'T) program. The total cost of the installed power and antenna is in the range of $\$ 40 \mathrm{M}$, or approximately $\$ 2$ per watt. This assumes $R \& D$ on the antenna assembly and on required improvements to the transmitter, which are accepted as components of the multiple-purpose $R \& D$ effort in the Development and Technology Division (D\&T) of the Office of Fusion Euergy (OFE) 
in the Department of Energy.

Seven CIT ports have been allocated to heating. If increased power is required relative to the baseline ( $20 \mathrm{MW} \mathrm{ICH})$, then two more ports can be assigned to ICH. The resulting system has $28 \mathrm{MW}$ at the plasma, and 14 FMIT units. Increasing ICH power beyond $28 \mathrm{MW}$ will require increased power density at the launchers. Such an increase is not planned.

\subsubsection{Alternate}

The choice of ECH was influenced by consistently successful heating in tokamaks, including high- $c_{-}$observations in Doublet-III-D, ${ }^{7}$ and by extremely high power densities for transmission ( $100 \mathrm{MW} \mathrm{m}^{-2}$ ), and by positive prospects for developing powerful sources in the tange of $300-600 \mathrm{GHz}$ as required for the high-field CIT. High power sources of millimeter radiation meeting CIT requirements have not been developed yet, but enthusiasm for ECH exists because the power can be launched in vacuum, some distance from the plasma, and because coupling is insensitive to plasma density.

Two technologies are receiving large R\&D funding from the OFE in the ECH area: the gyrotron and the Induction accelerator Free Electron Laser (IFEL). The OFE Development and Technology Division (D\&T) supports the gyrotron, and OFE Confinement Systems Division (CS) supports the IFEL. The IFEL project depends on spin-off from a weapons program. Plans on the gyrotron and the IFEL show development of ECH to a proof-of-principle in the early 1990's. The plan [or CIT shows a decision in the same period of time on whether one, or even both, methods should be included in the CIT project. Until that decision, the option of installing 10 to $30 \mathrm{MW}$ of ECH power is being maintained in the design process.

Preliminary cost studies show that gyrotron and IFEL implementations can be as low as \$4 per watt of tf power if a building is available. R\&D is not included in such an estimate. Use of an existing high-voltage de power system, such as the TFTR neutral beam power supply, can lower implementation costs by approximately $\$ 2$ per watt of $\mathrm{rf}$."

The CIT design goal for ECH is $15 \mathrm{MW}$ per port, averaging nearly $40 \mathrm{MW}$ $\mathrm{m}^{-2}$, so that $30 \mathrm{MW}$ is coupled through only two ports.

\section{$2.3 \quad$ Strategy}

CIT strategy regarding heating methods is as follows: 
1. The plan for CIT shows $20 \mathrm{MW}$ of $\mathrm{ICH}$ available at the beginning of operations.

2. Design of CIT and the ICH system is proceeding in accordance with this plan.

3. Design of the ECH systems is proceeding at the conceptual level. CIT design is proceeding with the guideline of keeping both ECH options open, based on the ECH design.

4. Four years or so prior to completion of the tokamak, CIT plans a decision on how to proceed with respect to ICH and ECH. 


\section{Design descriptions}

\subsection{ICH system}

\subsubsection{Physics}

The heating mode chosen for ICH is minority ${ }^{3} \mathrm{He}$ in a DD or DT plasma, owing to the high absorptivity and successful heating in past experiments. In a heated DT plasma, tritons can receive energy directly through second harmonic resonance once the $\beta$ is sufficiently high. At the CIT densities, the single-pass absorption rates are expected to be high, even if the exact location of cyclotron resonance is midway from plasma center to edge. Fast particles are not expected to be induced by the of fields due to the short equilibration times.

The frequency corresponding to $11 \mathrm{~T}$ is $105 \mathrm{MHz}$, including a $5 \%$ reduction from the nominal resonant formula to account for some improvement predicted for heating slightly outside the major radius. To allow heating over a range of fields, the rf source and transmission system is specified to cover $60-110$ $\mathrm{MHz}$ with a tuning time measured in hours.

\subsubsection{Antenna}

Coupling to the ICH waves is planned through inductive loops protected from the plasma by limiters or recesses in the vessel surface, or possibly both. Current elements in two toroidal locations in each port will allow spectral control, in particular to emphasize high $k_{\|}$most often associated with low impurity production. The loops are split in the poloidal direction to reduce voltages in the existing engineering design. ${ }^{10}$ Each loop is fed at the end, so eight coaxial transmission lines are mated to the flange on each of five ports. The resulting layout suffers from the crowding of components, so the option of two curreut loops separated toroidally will be studied. Recent experience with such loop couplers is consistent with the CIT goal of $4 \mathrm{MW}$ through each port of $0.4 \mathrm{~m}$ by $1.0 \mathrm{~m}$, making the net power density $10 \mathrm{MW} \mathrm{m}^{-2}$.

Disruptions can exert large forces on the Faraday shields and currentcarrying straps of the antenna, thereby leading to a difficult design problem, in large part owing to uncertainty in the behavior of the disruption. Heat loads from plasma and radiation are also somewhat uncertain, but it is clear that the goat of inertial cooling will be difficult to accomplish for a full ten seconds in a full-power, ignited experiment. 


\subsubsection{Source}

Ten modified FMIT units, each equipped with an upgrade to the EIMAC $\mathrm{X}-2242$ power tetrode, will supply the if power. To account for transmission and coupling losses, each unit is asked to provide $2.6 \mathrm{MW}$ at $105 \mathrm{MHz}$. Such an achievement has been judged to be possible with development effort. A joint effort of General Atomics, Varian, and the Japan Atomic Energy Research Institute has made progress in this direction.

\subsection{ECH physics}

For ECH, the ordinary mode with $\mathbf{E}_{\boldsymbol{r}} \| \mathbf{B}_{0}$ is selected becnuse it can be Inunched from the outside ports if the frequency is at or above the central cyclotron frequency. Good accessibility for fundamental harmonic waves is calculated up to a meximum density of $8 \cdot 10^{30} \mathrm{~m}^{-3}$, and absorption is strong when the wave crosses the cyclotron-resonant layer and $T_{\varepsilon}>1 \mathrm{keV}$ and $n_{e}>$ l. $10^{20} \mathrm{~m}^{-3} \cdot 1$

For ECH it may be that the development of a tunable, higlt-average-power source is impractical or impossible for frequencies in the desired range of $\mathbf{3 0 0}$ $\mathrm{GHz}$ ( $600 \mathrm{GHz}$ for second harmonic). Methods of heating the plasma over a range of fields are being investigated through modelling calculations. A very simple technique is to arrange for resonance to be outboard of center at the highest feld, and inboard of center at reduced field. More fexibility can be obtained by using the Doppler shift to heat particles of a given parallel velucity at locations that are not at cyclotron resonance. This would require a launching surface that can be angled either for different discharges, or as a function of time in a given discharge. ${ }^{11}$

The maximum density could be raised by going to second harmonic. In that case the extraordinary mode is preferred because of the stronger interaction with the electrons than the ordinary mode. In the case of a pulsed source of microwave power, such as the IFEL, nonlinear effects may reduce absorption, or cause other problems. Calculations on these matters suggest that no practically important effects will emerge, but experimental verification is planned. ${ }^{12}$

\subsection{Gyrotron-based implementation}

The power source is taken to be a nominal $1 \mathrm{MW}$ gyrotron tube operating at a frequency in the neighborhood of $300 \mathrm{GHz}$. Step-tuning is desirable ovet a range $200-310 \mathrm{GHz}$, but fixed-frequency is assumed. 
The TFTR neutral beam power supply is assumed to provide prime power, up to a maximum of $\mathbf{3 0}$ total $\mathrm{MW}$ of $\mathrm{rf}$ from 30 tubes. The existing transformers must be rewound in this high-power case. Other assumptions are $32 \%$ tube efficiency at $90 \mathrm{kV}$ and $35 \mathrm{~A}$, and $90 \%$ transmission.

Transmission from if source to plasma is a major problem in the case of these high frequencies. A corrugated circular waveguide operating in the $H E_{1 t}$ mode has been used at $60 \mathrm{GHz}_{+}$and may be applicable at $300 \mathrm{GHz}$. A purely optical transmisoion may be required, however, at some cost in space allocation. In each case, a beam combiner resentbling a Cassegrain telescope has been studied as a method to transport energy quasi-optically from the neighborhood of the gyrotrons, turning corners with spherical mirrors as the energy approaches the tokamak. Under this method, a window exists far from the tokamak at each gyrotron and another window exists at the tokamak vacuum boundary in the basement of the installation. ${ }^{3,13}$ Whatever the transmission method, pure mode output from the gyrotron tube and extensive transmission technology development will be required.

R\&D ie an important element in the gyrotron program. The problem is to develop and test sources and transmission systems at the $M W$ level, and in the frequency range of $300 \mathrm{GHz}$. The general approach is that Varian Associates will continue, from a base of 100 gyrotrons now sold, toward $\mathrm{cw}$ tubes at $\mathbf{5 0 0}$ kW and $1 \mathrm{MW}$ at frequencies of $140 \mathrm{GHz}$ and $110 \mathrm{GHz}$. The use of $110 \mathrm{GHz}$ tubes on a $2 \mathrm{MW}$ installation at General Atomics' Doublet-III-D tokamak will provide extra enthusiasm for concrete achievements in this area. In parallel with the work at Varian, the MIT Coherent Sources group at the Plasma Fusion Center is making progress on a megawatt-relevant, microsecond-pulsed gyrotron tube which uses a whispering gallery resonent cavity in a very high mode to minimize ohmic losses. Some recent short-pulse achievements are 925 $\mathrm{kW}$ at $148 \mathrm{GHz}$ with $20 \%$ efficiency, ${ }^{14}$ and step-tunability over $140-328 \mathrm{GHz}$ in the $400 \mathrm{~kW}$ range." Given success at Varien and MIT, it is conceivable that MW-level, $300 \mathrm{GH}$ s tube could be lannched before the mid-1990's in conjunction with a major ECH effort on CIT.

\subsection{IFEL implementation}

An IFEL starts with an electron beam in the dozen-MeV, several $k A$ range, which is passed through a wiggler structure at very low duty factor. The key advantages of this method are the potential tunability over $\approx 10 \%$ by operating within the gain curve, and over factors of two by adjusting the roltage and parameters of the different wiggler. A 10-40 MW implementation has been 
studied for CIT, concluding that the accelerators and wigglers could be placed in the TFTR 'mock-up' building. 16-18

The GW pulses (lasting dozens of nanoseconds) preclude use of a window, so the tokamak vacuum extends through the IFEL accelerator and wiggler, relalively far from the plasma. Transport of the microwave beam will require development, as with the gyrotron. Although beam efficiency is expected to be ligh - in the range of $30 \%$ - the wall plug efficiency may be in the range of $10 \%$ because of various losses in power conversion.

R\&D for the IFEL approsch is proceeding at the LLNL in the Microwave Tokamak Experiment (MTX). ${ }^{13}$ Plans call for single pulse experiments this year, and steady improvements leading up to $2 \mathrm{MW}$ for 0.5 seconds at 250 BHz before the mid-1990's. Experimental topics are current drive and MHD control, in addition to heating. A significant achievement on MTX in the direction of controlling disruptions would certainly lead to the consideration of the IFEL for that purpose on CIT.

\section{Acknowledgments}

This work was funded by the United States Department of Energy, Office of Fusion Energy, under conlrect DE-AC02-76-CHO-3073. Many others contributed to the considerations presented here, including: T. G. Brown, J. A. Casey, F. H. Coensgen, D. R. Cohn, P. L. Colestock, J. C. Commander, L. R. Grisham, H. Jory, R. R. Parker, M. Porkolab, D. B. Remsen, J. A. Schmidt, J. C. Sinnis, R. R. Stone, D. W. Swain, K. I. Thomassen, P. P. Woskov, J. J. Yugo.

This report is similar to a paper of the same name submitted to the IEEE Symposinm on Fuxion Engineering, Knoxville, Tennessee, October 3-5, 1989. 


\section{References}

'R. Parker, et al, "CIT Physics and Engineering Basis," paper IAEA-CN50/G-II-1, Twelfth International Conference on Plasma Physics and Controlled Nuclear Fusion Research, Nice, France, 12-19 October, 1988.

${ }^{2}$ D. P. Stotler and N. Pomphrey, "Pulse Length Assessment of CIT Designs," Princeton Plasme Physics Labotatory Report No. PPPL-2630, July 1989.

${ }^{3}$ D. W. Ignat, et al., "Assessment of Neutral Beams for the CIT," Princetont Plasma Physica Laboratory Report No. PPPL-TM-385, in press.

${ }^{4} D$. W. Ignat and M. Ono, "Ion Bernstein Wave Heating on the CIT," Princeton Plasma Physics Laboratory Report No. PPPL-2583, February 1989.

${ }^{5}$ B. J. D. Tubbing, J. Jacquinot, D. Stork, A. Tanga, " $H$-Mode in JET with ICH Alone," Nucl. Fusion (to be published).

"P. Colestock, et al., "ICH and ICH plus NBH Experiments in TFTR," in Radio-Frequency Power in Plasmas - AIP Conference Proceedings 190, Eighth Topical Conference, Irvine, CA, R. McWilliams, ed., published by the American Institute of Physics, New York, 1989.

${ }^{7} \mathrm{~J}$. Lohr, et $a l .$, "Observation of $H$-Mode in D-III-D with ECH," Phys. Rev. Lett. 60, 2630 (1988).

J. Casey, P. Woskot and D. Cohn, "Review of Basic System and Recent Studies for Gyrotron ECRH on CIT," CIT/MI'T report of 16 January, 1989 (unpublished).

'J. Stevens, et al., "Metal Impurity Behevior during ICH on TFTR," in RadioFrequency Pows in Plamms - AIP Conference Proceedings 190, Eighth Topical Conference, Irvine, CA, R. MeWilliams, ed., 1989, ibid.

${ }^{10} \mathrm{R}$. H. Goulding, et al., "ICRF Autenna Designs for CIT and Alcator CMOD," in Radio-Frequency Power in Plasmas - AIP Conference Proceedings 190, Eighth Topical Conference, Irvine, CA, R. McWilliams, ed., 1989, ibid.

${ }^{11}$ M. Porkolab, et al., "ECH-Assisted Startup anu Heating in the CIT," in Radio-Frequency Power in Plasmas - AIP Conference Proceedings 190, Eighth Topical Conference, Irvine, CA, R. McWilliams, ed., 1989, ibid. 
${ }^{12}$ E. B. Hooper, ed., "Microwave Tokamak Experimental Plan," Lawrence Livermore National Laboratory Report LLL-PROP-00202, May 1989 (unpublished).

${ }^{13}$ J. Casey and P. Woskov, "Feacibility Study of Combined Transmission Line," $\mathrm{CIT} / \mathrm{MII}$ memo of 24 October 1988 (unpublished). A related article is: J. Casey, P. Woskov, D. Cohn, R. Temkin, "Problems in Long Path High Power MM Wave Transmiasion for Gyrotron ECH in Tokamaks," symposium on Progress in Electromagnetic Research, Boston, 25 July 1989, (unpublished).

${ }^{11 K}$. E. Kreischer, et al., "Experimental Study of A High Frequency Megawatt Gyrotron Oscillstor," Ml'T report PFC/JA-89-33, July 1989, Phys. Fluids $B$ (to be published).

${ }^{15}$ K. E. Kreischer, et al., "Operation of a MW' Gyrotron in the Sub-mm Wave Region," to be publisted.

${ }^{16}$ R. A. Jong and R. R. Stone, "Preliminary Design of an IFEL Amplifier for Plasma Heating in CIT/I'I'ER," Lawrence Livermore National Laboratory report UCRL-99595, 15 September, 1988.

${ }^{17}$ R. R. Stone, et al., "An FEL-Based Microwave System for Fusion," Lawrence Livermore National Laboratory report UCRL-100851, J. Fusion Energy (to be published).

${ }^{14} \mathrm{R}$. Stone, "IFEL Options for $40 \mathrm{MW}, 280 \mathrm{GHz}$, Lawrence Livermore National Laboratory memo JCID-21705, 12 May 1989 (unpublished). 